\title{
START Proteins in Epilepsy
}

\author{
Sang Pil Yoon \\ Jeju National University \\ Republic of Korea
}

\section{Introduction}

Epilepsy is a condition with seizures by abnormal, recurrent, and excessive discharges from neurons. Observations in experimental models of epilepsy are providing a better understanding of the mechanisms of seizure-induced excitotoxicity, neuroprotective responses and neurogenesis (Naegele, 2007). Excitotoxic neurodegeneration can be investigated in epilepsy models employing kainic acid or pilocarpine. Each model results in distinctive patterns of degeneration within the hippocampus through kainate subclass of glutamate receptors or cholinergic receptors, respectively. Kainic acid triggers neuronal injury directly by binding to kainate receptors on mossy fiber endings and on the principal neurons of the hippocampus. Pilocarpine causes cell death of a majority of the nonprincipal neurons of the hilus and CA1 pyramidal cells.

Intensive researches have shown that some steroids display neuroprotective properties in various diseases including epilepsy (Wojtal et al., 2006; Biagini et al., 2010). The steroids can be classified into 'neuroactive steroids' and 'neurosteroids'. The term 'neuroactive steroids' may be synthesized both in the nervous system and in the endocrine glands such as gonads, adrenal glands and placenta, and exert their effects on neural tissue. 'Neurosteroids', the concept derived from Baulieu (1997), are a family of steroid hormones including pregnenolone, dehydroepiandrosterone, progesterone and their derivatives. Neurosteroids are synthesized in the nervous system de novo from cholesterol and accumulated in the nervous system independently of classical steroidogenic gland secretion rates.

Neurosteroids act in the nervous system in an autocrine/paracrine configuration (PlassartSchiees \& Baulieu, 2001). They may regulate gene expression by binding to nuclear receptors or affect neurotransmission through action at membrane ion-gated and other neurotransmitter receptors. In addition, they affect neuronal growth, survival and differentiation, cause regression of neuritic extensions before they have established contact with other neurons or glia and protect neurons from death. Modulatory roles of neurosteroids include $\gamma$-aminobutyric acid (GABA), N-methyl-D-aspartate, nicotinic/muscarinic cholinergic, serotonin, kainite and glycine receptor functions.

Neuroprotective properties of neurosteroids have been revealed and gained particular attention in the treatment of diseases where neurodegeneration is predominant, including epilepsy (Naegele, 2007; Biagini et al., 2010). Seizures not only cause cell death, but also elicit neuroprotective responses in injured neurons and glia. Seizures trigger the release of neuroactive steroids that impair hippocampal neuron survival in excitotoxicity. Increased neurosteroid synthesis, presumably occurring in glial cells during epileptogenesis, delays 
the appearance of recurrent spontaneous seizures in temporal lobe epilepsy. In this chapter, various molecules connected with neurosteroidogenesis are reviewed in order to provide further understanding on epilepsy.

\section{Neurosteroids and lipid/cholesterol}

Lipids have broad information carrying function in the central nervous system (CNS) as both ligands and substrates for proteins (Adibhatla \& Hatcher, 2008). Lipid metabolism may be important for the CNS, as this organ has the highest concentration of lipids next to adipose tissue. The crucial role of lipids in tissue physiology and cell signaling is demonstrated by the many neurological disorders, and altered lipid metabolism is also believed to be a key event which contributes to CNS injuries. Lipids serve not only structural components of the cell membrane but also precursors for various second messengers. Especially, cholesterol is an important regulator of lipid organization and the precursor for steroid biosynthesis.

The first step in the biosynthesis of neurosteroids is the conversion of cholesterol to pregnenolone (Wojtal et al., 2006; Biagini et al., 2010). This reaction is catalyzed by the cytochrome P450 cholesterol side-chain cleavage ( $\mathrm{p} 450 \mathrm{scc}$ ) in successive chemical reactions. First and foremost, transport of cholesterol to the mitochondria is a prerequisite for neurosteroidogenesis. Cholesterol is not only needed for the growth and remodeling of neuronal and glial membranes, but also is an important regulator of lipid organization and the precursor for steroid biosynthesis (Adibhatla \& Hatcher, 2008). The majority of cholesterol in the brain is derived from de novo synthesis in the neurons, astrocytes predominantly, and oligodendrocytes. Cholesterol is then secreted via transport molecules, taken up by lipoprotein receptors on neurons and internalized to the endosome/lysosome system.

Cholesterol is transported to mitochondria by Niemann-Pick C1 (NPC1) protein where the neurosteroids are synthesized via the rate limiting intermediate, pregnenolone (Adibhatla \& Hatcher, 2008). NPC1 protein is involved in transport of lipids, particularly cholesterol, from the late endosome/lysosome. Deficiency in the protein results in lysosomal accumulation of cholesterol and other lipids. Then, proteins located in the mitochondrial membranes, such as peripheral benzodiazepine receptor (PBR) and steroidogenic acute regulatory protein (StAR), allow cholesterol to cross the hydrophilic intermembrane space (Lavaque et al., 2006). In the mitochondria, the neurosteroids are synthesized from the cholesterol via the rate limiting intermediate pregnenolone.

Observations are providing a better understanding of the transport of cholesterol by NPC1, PBR or StAR (Adibhatla \& Hatcher, 2008). Niemann-Pick disease type C is due to mutations in either the NPC1 or NPC2 genes, resulting in defective cholesterol transport and cholesterol accumulation. The PBR, which might function as a cholesterol channel, is essential for the delivery of cholesterol to the inner mitochondrial membrane and the synthesis of steroids. However, it should be noted that these molecules are not the only molecules implicated in the metabolism of cholesterol and little attention has been paid to StAR-related lipid transfer (START) domain-containing proteins (START proteins).

\section{START proteins}

START domain is an evolutionary conserved protein module of approximately 210 amino acids (Soccio \& Breslow, 2003; Alpy \& Tomasetto, 2005; Lavigne et al., 2010). START domain 
is conserved through in plants and animals and serves as a versatile binding interface for lipids. The crystal structures of solved START domain reveal a conserved 'helix-grip' fold, in which a central antiparallel $\beta$-sheet is gripped by N-terminal and C-terminal a-helices, the latter being closely packed above the nine-strand curved $\beta$-sheet.

In humans, START domains are found in 15 distinct proteins and can be classified by 6 subfamilies (Table 1). Mammalian START proteins have diverse expression patterns and can be found free in the cytoplasm, attached to membranes or in the nucleus. START proteins implicate in intracellular lipid transport, lipid metabolism, and cell signaling events. Mutation or mis-expression of START proteins is linked to pathological processes, including genetic disorders, autoimmune diseases and cancer. Nevertheless, START proteins have not been extensively studied in the nervous system except 2 proteins, StAR and StarD6.

\begin{tabular}{llll}
\hline Group & Other names & Subcellular localization & Gene \\
\hline StarD1 & StarD1/StAR & Mitochondria & $8 \mathrm{p} 11.2$ \\
& StarD3/MLN64 & Late endosomes & $17 \mathrm{q} 11-\mathrm{q} 12$ \\
\hline StarD4 & StarD4 & Cytosol and Nucleus & $5 \mathrm{q} 22.1$ \\
& StarD5 & Cytosol and Nucleus & $15 \mathrm{q} 26$ \\
& StarD6 & Nucleus & $18 \mathrm{q} 21.2$ \\
\hline StarD2 & StarD2/PCTP & Cytosol & $17 \mathrm{q} 21-\mathrm{q} 24$ \\
& StarD7 & $?$ & $2 \mathrm{q} 11.2$ \\
& StarD10 & Cytosol and Nucleus & $11 \mathrm{q} 13$ \\
& StarD11/CERT & Cytosol and Golgi & $5 \mathrm{q} 13.3$ \\
\hline RhoGAP & StarD8 & $?$ & Xq13.1 \\
& StarD12/DLC-1 & Plasma membrane & $8 \mathrm{p} 22$ \\
& StarD13/DLC-2 & Cytosol & $13 \mathrm{q} 12-\mathrm{q} 13$ \\
\hline Thioesterase & StarD14/BFIT & $?$ & $1 \mathrm{p} 32.3$ \\
& StarD15/CACH & Cytosol & $5 \mathrm{q} 14.1$ \\
\hline StarD9 & StarD9 & $?$ & $15 \mathrm{q} 15.1-\mathrm{q} 15.2$ \\
\hline
\end{tabular}

Table 1. Phylogenetic group and 15 human START proteins quoted from Alpy \& Tomasetto (2005) with slight modification. BFIT, brown fat-inducible thioesterase; $\mathrm{CACH}$, cytoplasmic acetyl-CoA hydrolase; CERT, Goodpasture-antigen-binding protein $\triangle 26$; DLC, deleted in liver cancer; MLN64, metastatic lymph node 64; PCTP, phosphatidylchoine transfer protein; RhoGAP; Rho-GTPase-activating-protein-domain.

\subsection{Subcellular characteristics of StAR and StarD6}

StAR, the prototype of START proteins, is first characterized in murine MA-10 Leydig tumor cells that luteinizing hormone induces the expression of StAR and ultimately results in increased synthesis of pregnenolone (Clark et al., 1994). Its function has been extensively studied in classical steroidogenic tissue such as adrenal gland and ovary, and it has been shown to be involved in the intramitochondrial trafficking of cholesterol (Stocco, 2001). In steroidogenic cells, StAR-mediated delivery of cholesterol to inner mitochondrial membrane is a hormonally regulated step and StAR expression is tightly regulated (Stocco, 2001; Stocco et al., 2005).

StarD6 is originally reported in male germ cell-specific protein of StarD4 group of START proteins (Soccio et al., 2002; Gomes et al., 2005). It is immunolocalized only in the nuclei of germ cells, not in the interstitial cells of Leydig, and may have a pivotal role in 
spermatogenesis rather than in steroidogenesis. StarD6 is detected in the nucleus of mature sperm cells, where it could interact with transcriptional machinery in a lipid-dependent manner. Since lipids play an important role in sperm capacitation and function, StarD6 might regulate lipid movement within the sperm cell membrane.

StarD6 in the Leydig cells has also been reported under perinatal hypothyroidism while germ cell-specific immunoreactivity is delayed (Chang et al., 2007a), which means that StarD6 also might play a role in the steroidogenesis under certain conditions such as testosterone deficiency. In this context, StarD6 might interact with mitochondrial membranes just similar to that of StAR (Bose et al., 2008). StarD6 has a protease-sensitive C-terminus similar to that of StAR and exhibits a steroidogenic activity equal to StAR. Recently, this question is ascertained in the nervous system by morphometry (Chang et al., 2010).
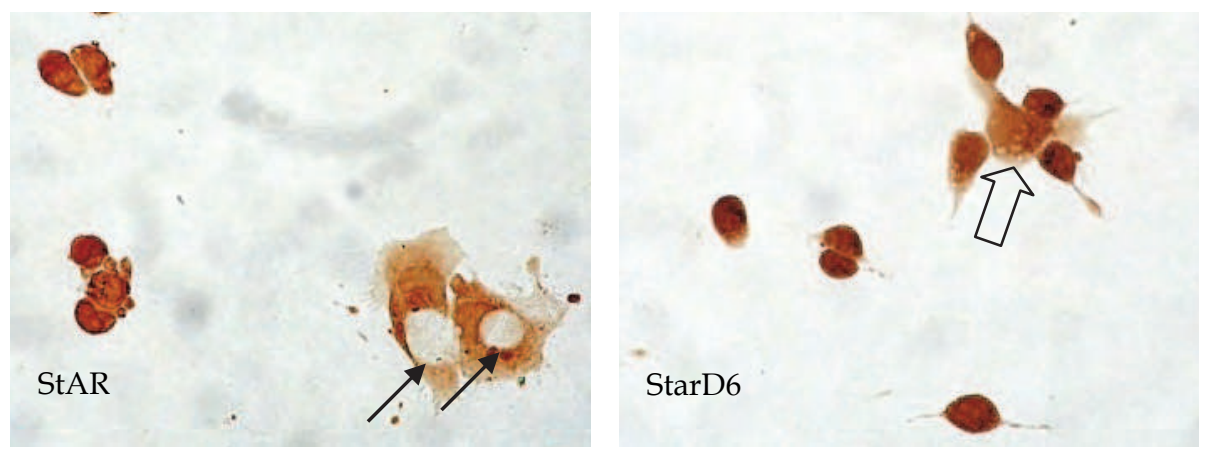

Fig. 1. Micrographs of StAR and StarD6 in cultured dopaminergic neurons (SK-N-SH cell line). StAR is localized in the cytoplasm (arrows) but StarD6 loses the localization in nucleus (open arrow) according to maturation. Original magnification X40. (quoted from Chang et al., 2010)

Morphological aspects of StarD6 are significantly different from those of StAR in cultured neuronal and glial cells. Cultured cells are dopaminergic neuronal cells (SK-N-SH) and glioma cells, M059-K and M059-J, which M059-J lack DNA-dependent protein kinase (DNA-PK) activity while M059-J express normal levels of DNA-PK. StAR tends to localize in the cytoplasm but StarD6 showed a weak intensity in the nucleus according to neuronal maturation (Fig. 1), while both are immunolocalized in the nuclei as well as the cytoplasm in glioma cells. The number of StAR and StarD6 immunopositive cells is significantly different in SK-N-SH and M059-K and M059-J, respectively. Especially, the immunoreactivities of StarD6 in glioma cells are considerably changed by the presence of DNA-PK, while there is no significance in StAR (Fig. 2).

The morphometric results with StarD6 in glioma cells have meaning, since oxidative DNA damage by excitotoxicity may activate DNA repair proteins in affected neurons (Naegele, 2007). The number of StarD6 is significantly decreased in the glioma cell line which lacks DNA-PK activity. DNA-PK plays a role in nonhomologous end joining (NHEJ) against DNA damage, which comprises of catalytic subunit of the DNA-PK (DNA-PKcs), Ku70, and Ku80 (Martin, 2008). NHEJ system is activated by severe forms of DNA damage, DNA doublestand breaks. Increasing evidences implicate DNA-PK in neuroprotective properties. Experimental DNA-PK null mice show increased excitotoxicity and result in augmented 
apoptosis (Culmsee et al., 2001; Neema et al., 2005). It suggests that the activity of StarD6 in the nucleus might depend on the DNA repair system in preventing DNA damage.

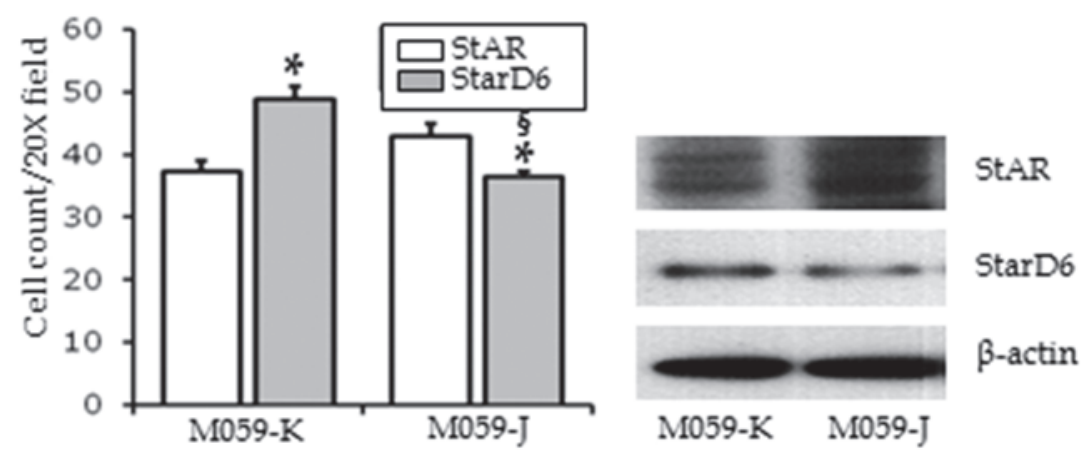

Fig. 2. Histographs of StAR and StarD6 in cultured glioma cell lines. M059-J cells lack DNAdependent protein kinase (DNA-PK) activity, while M059-K cells express normal levels of DNA-PK (acquired from American Type Culture Collection, ATCC, Rockville, MD, USA). The number of immunopositive cells was significantly different by StAR or StarD6 in the M059-K and M059-J, respectively ( $\left.{ }^{*} \mathrm{p}<0.05\right)$. Unlike StAR, the immunoreactivities of StarD6 were considerably changed by the presence of DNA-PK $(\S p<0.05)$, and it was also demonstrated by Western blot analysis. Data are quoted from Chang et al., 2010 (left) and unpublished data (right).

\subsection{Distribution of StAR and StarD6 in the nervous system}

StAR appears to be widely distributed throughout the brain, although different levels of expression have been detected between different brain areas including human (Furukawa et al., 1998; King et al., 2002; Sierra et al., 2003; Sierra, 2004; Lavaque et al., 2006). High levels of StAR mRNA expression have been detected in the cerebral cortex, hippocampus proper, dentate gyrus, olfactory bulb, and cerebellar cortex. In general, there is a good agreement between those from in situ mRNA localization and data from immunohistochemical studies. This may implicate that steroidogenesis is a generalized process in the nervous system.

StAR immunoreactivity has been detected in several neuronal populations, in ependymocytes and in some astrogial cells in the brain (Lavaque et al., 2006). StAR expression, however, seems to be restricted to very specific neuronal and astroglial populations in each brain area. Strong StAR immunoreactivity is observed in the soma of large neurons, which are characterized by high mitochondrial content. Although StAR is predominantly expressed by neurons in the CNS, astrocytes also express STAR and is known to synthesize neurosteroids from cholesterol. StAR may contribute to the regulation of cell proliferation in the nervous system, since steroids are known to affect neuronal and glial differentiation (Lavaque et al., 2006). Important changes in the pattern and/or level of expression of StAR occur in different brain areas during postnatal development (Kim et al., 2002; Sierra et al., 2003). For instance, in the cerebellar cortex Purkinje cells express StAR as well as have an active steroidogenic activity, in particular during the developmental period (Tsutsui et al., 2003). Changes of StAR in the developing cerebellar cortex may therefore be related with the differentiation of Purkinje cells. Important changes in the expression of StAR occur as well during brain aging. In 24-month-old rats StAR 
immunoreactivity is increased in hippocampal and cortical neurons compared with young animals (Sierra et al., 2003), while decreased in the aged cerebellum (Lavaque et al., 2006). It is unknown what is the physiological significance of these changes in StAR expression in the brain and whether they are associated with differences in steroidogenesis.

Furthermore, StAR is colocalized in the same neural cells with p450scc and with other steroidogenic enzymes (Furukawa et al., 1998; King et al., 2002). It may implicate that StAR as a transport protein may participate in neurosteroidogenesis in the CNS. Strikingly, no deficiency in brain function or structure was reported either in StAR knockout mice or in congenital adrenal hyperplasia patients (Sierra, 2004). The brain may have StARindependent mechanisms of mitochondrial cholesterol transport. It should be noted that other proteins that contain the START domain might be involved in steroidogenesis and that their regional patterns of expression in the brain remain to be determined.

\begin{tabular}{lll}
\hline & StAR & StarD6 \\
\hline Cerebrum & Neuron (layers V-VI) $>$ >glia & Neuron (layers V-VI) >glia \\
\hline $\begin{array}{l}\text { Hippocampus } \\
\text { Cornu ammonis }\end{array}$ & Strata oriens/radiatum & Stratum pyramidale \\
Dentate gyrus & Mild granular layer & Granular layer \\
\hline Cerebellum & Purkinje cell $(+)$ & Purkinje cell $( \pm)$ \\
\hline
\end{tabular}

Table 2. Distribution of StAR and StarD6 in the nervous system. Although StAR and StarD6 can be detected in neurons and glia, they are primarily immunolocalized in the neurons of rodents. Unlike the cerebral cortex, they have compensatory distribution in the hippocampus and cerebellum. (According to Furukawa et al., 1998; King et al., 2002; Sierra et al., 2003; Sierra, 2004; Chang et al., 2007b, 2009, 2010)

Not much is known on the distribution, development and aging of StarD6 in the CNS (Table 2). StarD6 is seen in the cerebral cortex, cerebellum, hippocampus, spinal cord and dorsal root ganglia (Chang et al., 2007b, 2009), where the highest expression of StAR is reported (Sierra, 2004; Lavaque et al., 2006). Strong StarD6 immunoreactivity is observed in deeper layers of cerebral cortex, principal cell layers of hippocampus and substantia gelatinosa of spinal cord, but devoid in Purkinje cell layer of cerebellum and lower motor neurons of spinal cord. From morphological point of view StarD6 also immunolocalized in glial cells, but the characteristics are not determined properly.

StarD6 immunolocalization was mainly restricted to the nucleus, but cytoplasmic immunostaining frequently appeared in the principal neurons of hippocampus and spinal cord. This subcellular localization pattern is a distinctive feature of StarD4 group (Alpy \& Tomasetto, 2005; Soccio et al., 2005). But the characteristics of the immunostaining of the StarD6 in the cytoplasm and the nucleus remain largely undetermined. The existing data (Gomes et al., 2005; Chang et al., 2007a,2007b,2009,2010; unpublished data) are obtained with the rat-specific polyclonal anti-StarD6 antibody (raised from Dr. Soh's laboratory, Chonnam National University, Republic of Korea), and it should be confirmed by the monoclonal antibody.

\subsection{Changes of StAR and StarD6 in epilepsy}

\subsubsection{Generalized changes of StAR and StarD6 in epileptic hippocampus}

Neurosteroids can interact with various neurotransmitters (Joëls, 2009; Biagini et al., 2010). In particular, neurosteroids are well known to potentiate the actions of GABA via GABA 
receptors against stress. Neurosteroids not only facilitate inhibitory responses, but also reduce the slope of the field excitatory postsynaptic potential in the dentate gyrus toward more inhibition. Various neurotrophic factors, including hormones, alter cellular activity in all hippocampal subfields in a region-specific way (Joëls, 2009).

StAR has been related to neuroprotection (Sierra et al., 2003; Sierra, 2004). StAR mRNA and protein levels are transitorily increased following excitotoxic brain injury induced by the administration of kainic acid (Sierra et al., 2003). A fast and transient increase in StAR mRNA levels in the hippocampus was detected in the first 12 hours after injury. This was accompanied by an increase in StAR immunoreactivity in granular and hilar hippocampal neurons in the first 12-24 hours after the administration of kainic acid.

In addition, StAR immunostaining is more intense in neurons located in the strata oriens and radiatum of normal hippocampus. The pattern of distribution changed in the pilocarpine-induced epileptic hippocampus (Chang et al., 2010). StAR was immunolocalized in the stratum pyramidale as well as the strata oriens and radiatum 3 hours after pilocarpine treatment, while changed immunoreactivity in the dentate gyrus was not remarkable. But, the changes in distribution in the hippocampus proper did not discussed in kainic acidinduced epilepsy model (Sierra et al., 2003).

A strong increase in StAR immunoreactivity was observed in layers II and III of the pyriform cortex of kainic acid-injected animals (Sierra et al., 2003). However, in deeper cortical layers the cellular mortality was very high. Therefore, a large increment of StAR expression of hippocampal granular neurons after brain injury is especially resistant to the toxicity of kainic acid. The expression in dentate gyrus is related to neuroprotection in status epilepticus. Moreover, subgranular neurons have mitotic activity and increased StAR might affect on neurogenesis and differentiation in epileptic hippocampus.

StarD6 is acutely and transiently increased after excitotoxic brain injury also (Chang et al., $2009,2010)$. The changes are seen in the granular and polymorphic layers of the dentate gyrus and the CA subfields of the hippocampus 3 hours after pilocarpine treatment. In the CA1-2 areas, StarD6 immunoreactivity was increased in the strata lacunosum-moleculare as well as stratum oriens and stratum radiatum after the lesion. This increase in immunolocalization remained until 12 hours after experiencing epilepsy, and then slightly decreased up to 24 hours after epilepsy. StarD6 immunoreactivity remained in the pyramidal layer of the CA subfields, whereas the granular layer of dentate gyrus was not very intensely stained. In agreement with the distributional changes, a significant increase in StarD6 protein is detected 3 hour after brain injury with respect to the untreated animals and 24 hours after epilepsy.

Similar results in distribution and Western blot analysis are obtained in domoic acid-induced epilepsy model (unpublished data). Domoic acid is a kainic acid analogue and characteristically damages the hippocampus since hippocampus has well developed glutamate receptors (Coulter et al., 2002; Naegele 2007). Western analysis revealed significant change in the level of StarD6 in response to domoic acid, an increase being observed 4 hours after the epilepsy compared with normal hippocampus.

\subsubsection{Time-course of StAR and StarD6 after epileptic injury}

Neurosteroids are potent trophic and survival factors for the nervous system and the synthesis is related to the extent of p450scc induction. The p450scc enzyme is found in neurons, astrocytes, oligodendrocytes, and in activated microglial cells (Biagini et al., 2010). 

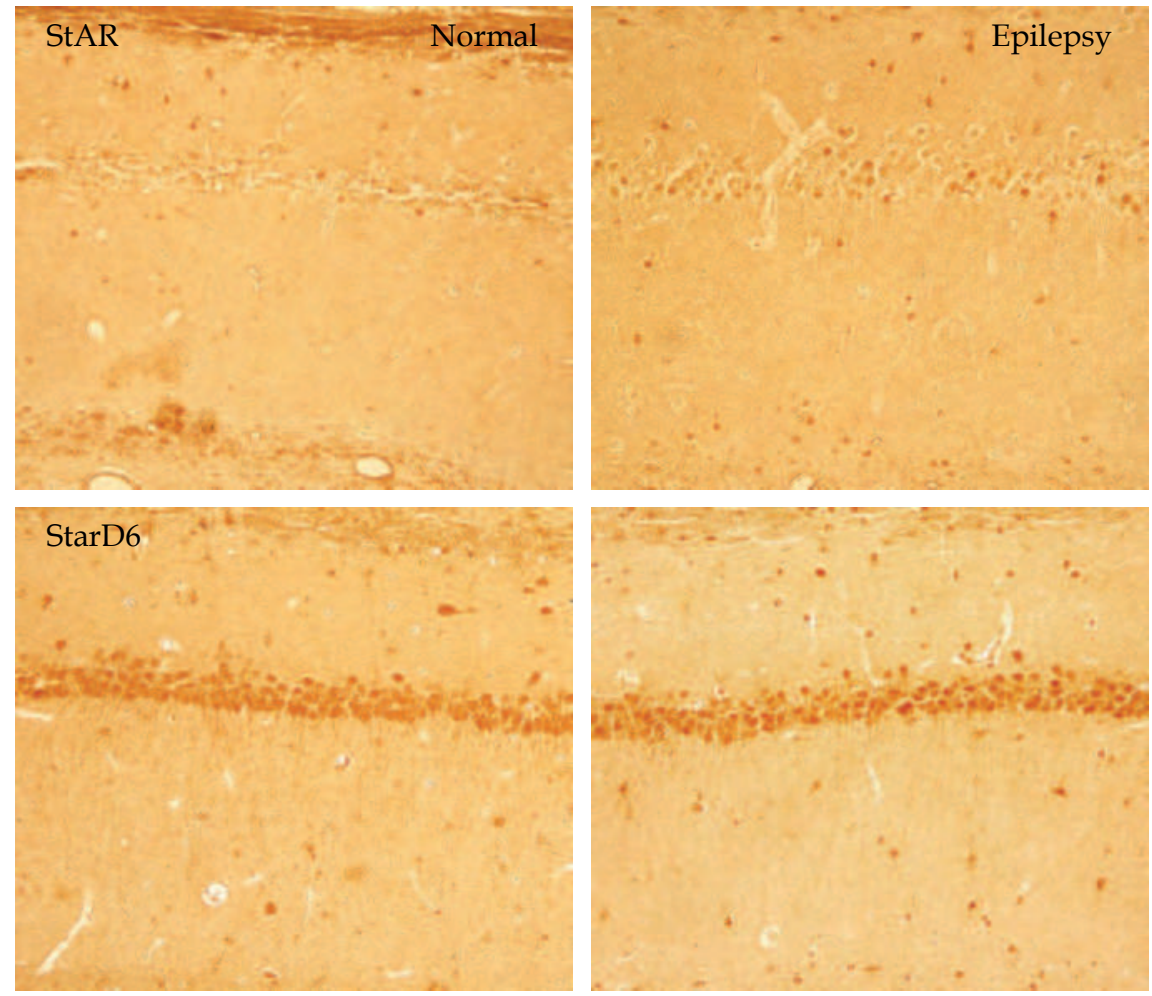

Fig. 3. Compensatory distribution of StAR and StarD6 in normal and epileptic hippocampus. StAR immunolocalization was seen in the strata oriens and radiatum while StarD6 in the stratum pyramidale in normal hippocampus. Augmented immunolocalization of StAR was observed in the stratum pyramidale and StarD6 in the strata lacunosummoleculare after pilocarpine-induced epilepsy. Figures are aligned by stratum pyramidale. Original magnification X20.

Neurosteroid levels can be altered as consequence of neuronal damage as well as of glial cell activation. A significant increase in p450scc both in neurons and glial cells is reported, but the neuron-specific changes were limited to the first few days after status epilepticus whereas those in glial cells were long-lasting. Therefore, neurosteroidogenesis is related to the extent of p450scc induction in glial cells consequent to status epilepticus.

Activation of various molecules connected with steroidogenesis after brain injury results in a significantly increased synthesis of neurosteroids. Interestingly the increase in StAR and StarD6 was restricted to neurons in epileptic hippocampus. An urgent increase of StarD6, even compared with StAR, after excitotoxic brain injury may be the first attempt to respond to primary neurodegeneration (Figure 4). Early induction of StarD6 as well as StAR is followed by later increase in the expression of various steroidogenic enzymes including p450scc (Furukawa et al., 1998; Garcia-Ovejero et al., 2002; Biagini et al., 2006).

The induction of StAR in neurons under neurodegenerative conditions is followed by the increase in the expression of PBR in glial cells (Lavaque et al., 2006). The induction of StAR and PBR has a different time course. StAR expression is increased within the first 
day after lesion while the increase in PBR expression is observed from 1 to 5 weeks after lesion. Therefore, StAR and PBR may participate in different phases of steroidogenesis after brain injury. The increased expression of these molecules may be the cause of the increase in the production of neurosteroids, such as pregnenolone. Changes in the expression of StAR and PBR may also result in the production of other neuroprotective steroids.

Meanwhile, the results with StarD6 suggest another aspect on neurosteroidogenesis. On the assumption that StarD6 participate in the regulation of the production of neuroprotective steroids, the responses of StarD6 might be coincided with the hypothesis that it is the first attempt to respond to epilepsy in accordance with brain injury resulting in a significant increased synthesis of neurosteroids (Di Michele et al., 2000). As a result, nuclear immunostaining and early response of StarD6 may regulate gene expression by binding to nuclear receptors (Mellon et al., 2001; Plassart-Schiess \& Baulieu, 2001). StarD6 can act as transcriptional machinery in a lipid-dependent manner and thus stimulate neurosteroidogenesis by activating StAR and other steroidogenic enzymes. But, direct relationship between StAR and StarD6 is not confirmed yet in the nervous system.

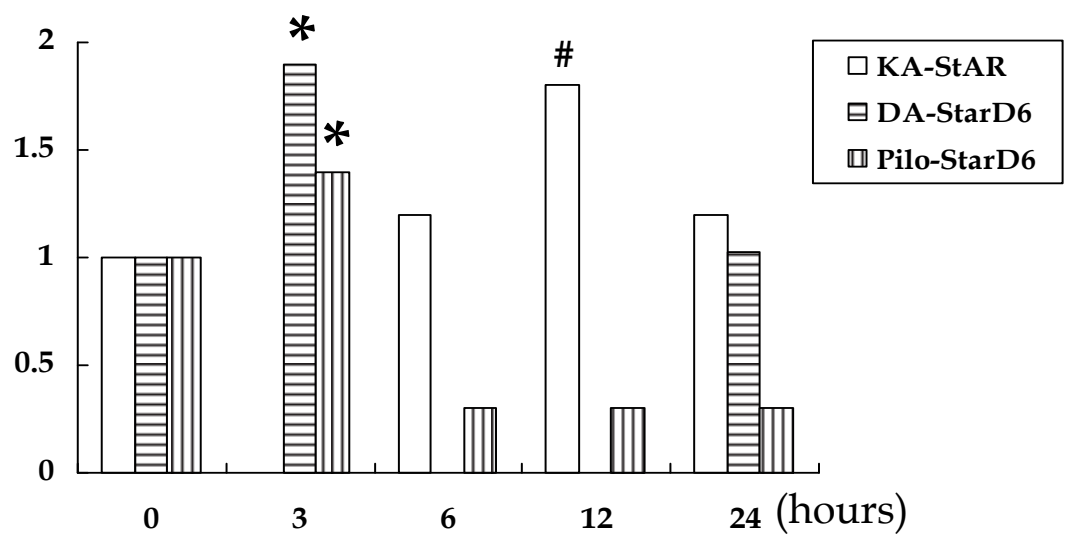

Fig. 4. Representative changes in StAR and StarD6 in the hippocampus after excitotoxic insult. The histogram shows relative densitometric values of StAR mRNA (Sierra et al., 2003) and StarD6 protein (Chang et al., 2009; unpublished data). The animals killed $12 \mathrm{~h}$ after kainic acid (KA) administration showed a significant increase in StAR mRNA levels. Considerable changes in StarD6 level in response to domoic acid (DA) or pilocarpine (Pilo) observed 3-4 hours after the epilepsy. (The densitometric results are modified from original manuscript)

\subsubsection{Area-specific changes of StAR and StarD6 in epileptic hippocampus}

Epilepsy could cause widespread neurodegeneration in CA1-3 pyramidal layers of the hippocampus (Naegele, 2007), but there is enough ground for controversy on the areaspecific degeneration of hippocampus. Epilepsy-related neurodegeneration is mainly limited to CA3 area of the hippocampus (Neema et al., 2005; Kajitani et al., 2006; Ma et al., 2006; Chuang et al., 2009; Zhang et al., 2009), while the neurons in CA1 area survive in 
various animal models (Marti et al., 2002; Dinocourt et al., 2003; Cavazos et al., 2004; Sanon et al., 2005; Wittner et al., 2005).

When rats are treated with kainic acid, a rapid and transient increase in StAR mRNA and protein was detected 12 hours after brain injury in the hippocampus (Sierra et al., 2003). A strong increase in StAR immunostaining was observed in the granular layer of dentate gyrus while the control hippocampus was not very intensely stained. The results do not provide effective involvement of StAR for steroidogenesis, but possible role in neuroprotection in neurons against excitotoxic injury. In this context, distribution of StAR changed in the pilocarpine-induced epileptic hippocampus (Chang et al., 2010). StAR immunostaining is localized in the strata oriens and radiatum of normal hippocampus, and in the stratum pyramidale as well in epileptic hippocampus. The changes in immunolocalization are reported in CA1-2 areas, not in the dentate gyrus.

Similar results on area-specific changes are reported with StarD6 in epileptic hippocampus (Chang et al., 2009, 2010; unpublished data). The StarD6 immunolocalizations are seen in the strata lacunosum-moleculare as well as strata oriens and radiatum after excitotoxic injury with pilocarpine and domoic acid, respectively. The changes in the strata correspond with distribution of steroidogenic enzymes such as p450scc (Furukawa et al., 1998; GarciaOvejero et al., 2002; Biagini et al., 2006). As a result, StarD6, StAR and p450scc might act in consecutive order following excitotoxic brain injury, result in neurosteroidogenesis.

In addition, the morphometric data (Chang et al., 2010) with StarD6 in DNA-PK deficient cells are in accord with the previous reports that lack of DNA-PK promotes apoptosis (Culmsee et al., 2001; Neema et al., 2005). It can be interpreted that the activity of StarD6 in the nucleus might depend on the DNA repair proteins. To investigate whether StarD6 is related with DNA repair system in nervous system, epilepsy model was established by domoic acid (unpublished data, Fig. 5). Contrary to the hypothesis based on previous reports, there was negative correlation between StarD6 and NHEJ (data not shown).

But the levels of apurinic/apyrimidinic endonuclease (APE, also called redox factor-1) and 8-oxoguanine DNA glycosylase (Ogg1) increased considerably at 4 hours after domoic acid injection. The responses are quite similar to previous reports with kainic acid (Quach et al., 2005; Jarrett et al., 2008) and that of StarD6 (Fig. 4). The results support the notion that oxidative DNA damage in neurons is primarily removed by base excision repair (BER) pathway rather than by NHEJ (Fishel et al., 2007). Despite activation of DNA repair systems after excitotoxic injury, the repair responses may not sufficient to overcome for extensive DNA damage.

The distribution of BER proteins revealed that transient increase of APE in strata radiatum and lacunosum-moleculare and Ogg1 in stratum pyramidale. Increased immunoreactivities of APE and Ogg1 diminished in the pyramidal cells of CA3 area 24 hours after domoic acid injection compared to CA1. In case of StarD6, the immunostaining was induced in the strata radiatum and lacunosum-moleculare after epilepsy and then normalized in the stratum pyramidale in CA3 as well as CA1. Neurodegeneration in CA3 pyramidal cells may be caused by the decreased BER activity after seizure since there was no area-specific change in StarD6 especially in CA3. StarD6 in the nucleus did not respond on DNA repair system after epilepsy, but immunolocalization of StarD6 as well as APE in the strata lacunosummoleculare may have significance where localized various steroidogenic enzymes (Furukawa et al., 1998; Patte-Mensah et al., 2003; Biagini et al., 2006; Wojtal et al., 2006; Chia et al., 2008). 

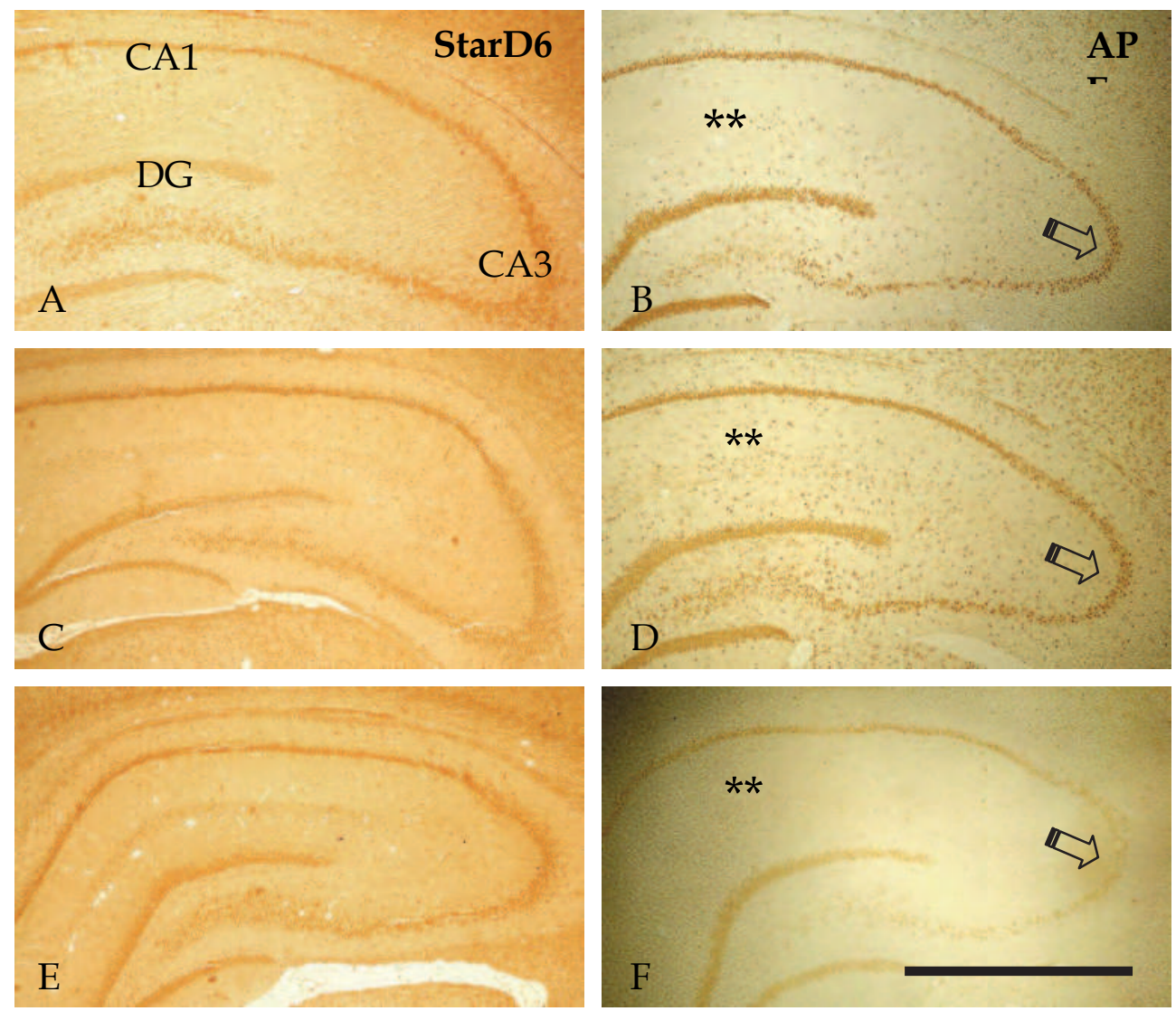

Fig. 5. Area-specific changes of StarD6 and APE in epileptic hippocampus.

Immunoreactivities of StarD6 and APE increased in the strata radiatum and lacunosummoleculare $\left(^{*}\right) 3$ hours after epilepsy (C and D) compared with control hippocampus (A and B) or 5 days after epilepsy (E and F). Note the changes in stratum pyramidale. Although StarD6 immunoreactivity remained in all subfields, the intensities diminished in CA3 (arrow) while those in CA1 were maintained. Scale bar $=1 \mathrm{~mm}$.

\subsubsection{Functional aspects of StAR and StarD6 in epileptic hippocampus}

StAR has been shown to be involved in the intramitochondrial trafficking of cholesterol (Alpy \& Tomasetto, 2005; Lavigne et al., 2010). Then, cholesterol is made available to the first enzyme of the steroidogenic pathway, p450scc, which transforms cholesterol into pregnenolone, the precursor for glucocorticoids, mineralocorticoids and sex steroids. StARmediated delivery of cholesterol to the inner mitochondrial membrane, where p450scc is located, is a hormonally regulated step, which is rate limiting in steroidogenesis.

The up-regulation of StAR expression after excitotoxic injury suggests that this protein may play a role in the adaptation of neural tissue to neurodegenerative conditions (Sierra et al., 2003; Sierra, 2004; Lavaque et al., 2006; Chang et al., 2010). That is, StAR in the dentate gyrus and hippocampus might be associated with neuroprotection and neurosteroidogenesis, 
respectively. Strikingly, no deficiency in brain function or structure was reported either in StAR knockout mice or in congenital adrenal hyperplasia patients (Sierra, 2004). The brain may have StAR-independent mechanisms of mitochondrial cholesterol transport.

It should be noted that StAR is not the only molecule implicated in the mitochondrial transport of cholesterol. PBR, which might function as a cholesterol channel, is essential for the delivery of cholesterol to the inner mitochondrial membrane and the synthesis of steroids. NPC1 is also involved in transport of lipids from the late endosome to mitochondria. Furthermore, there has been described a family of proteins which contain the START domain. These START domain proteins, such as StarD6, are related to steroidogenesis.

In some START proteins, the START domain probably functions in lipid sensing rather than in lipid transfer (Alpy and Tomasetto, 2005; Lavigne et al., 2010). StarD2/PCTP, StarD4, StarD5 and StarD10 are detected in the cytoplasm and nucleus, and StarD6 only in the nucleus. It is therefore possible that some START-containing proteins have nuclear roles and may even regulate transcription in a lipid-dependent manner. Structurally, the START domain contains a lipid-binding domain specific for diacylglycerol/phorbol-ester, the $\mathrm{C} 1$ domain (Brose and Rosenmund, 2002) and functions as a lipid-sensing domain providing a rapid way of regulating the catalytic activity, and thus modulates lipid metabolism.

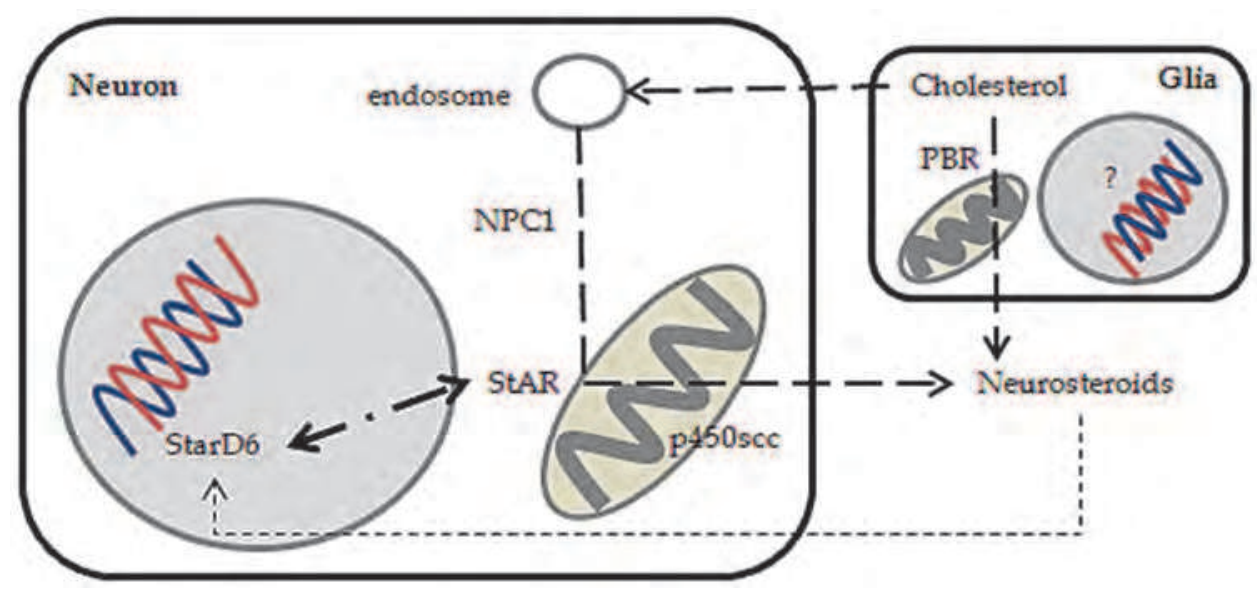

Fig. 6. Schematic representation of various molecules participating in neurosteroidogenesis. StAR and PBR is mainly localized in the outer mitochondrial membrane of neuron and glia, respectively, while p450scc acts in the inner mitochondrial membrane. Note StarD6 in the nucleus, which may interact with StAR for neurosteroidogenesis. Stimulus-dependent upregulation of START proteins appears as follows; StarD6 in a few hours and StAR in a day, while PBR and p450scc in a few weeks.

The changed immunolocalization of StarD6 in the strata lacunosum-moleculare might suggest a role of this protein as a regulator of transcription related to the cholesterol metabolism (Chang et al., 2009, 2010, unpublished data). Although StAR is a well known protein implicated in the steroidogenesis, StarD6 changed the localization from stratum pyramidale to the strata lacunosum-moleculare after excitotoxic injury. Another important change is the time course of responsiveness after epilepsy. The response of StarD6 arises 
from the nucleus with DNA repair proteins to respond to excitotoxic stimuli. StarD6 can function as a lipid-sensing domain and modulate neurosteroid synthesis.

There is growing evidence that cholesterol is of particular importance in development of Alzheimer's disease (Wojtal et al., 2006; Adibhatla \& Hatcher, 2008). In the patients' brains of Alzheimer's disease, a general trend was observed towards decreased levels of all steroids. The lower levels correlated with increased amounts of $\beta$-amyloid peptides and phosphorylated tau proteins. Increased level of StAR is reported in aged rats (Sierra et al., 2003) and in patients with Alzheimer's disease (Webber et al., 2006) compared with young animals and age-matched patients without dementia, respectively. It means that the expression of StAR hormonally regulated by the level of neurosteroids in the nervous system. Although the existing knowledge on StarD6 is still incomplete, it can be expected that StarD6 is also regulated by the levels of neurosteroids, cholesterol and lipid. Furthermore, StarD6 is localized in the nucleus and appears early response than StAR in epilepsy (Chang et al., 2009, 2010, unpublished data), results in stimulus-dependent expression of StAR follows after StarD6.

\section{Conclusion}

The START domain operates as a lipid exchange and/or a lipid-sensing domain. There are 15 mammalian proteins that possess a START domain, an evolutionary conserved protein module and they are involved in several different biological processes: lipid transfer, lipid metabolism and signal transduction. While the role of StAR in steroid hormone production has been demonstrated, StarD6 remain largely uncharacterized. Much less is understood about how its START domain specifically recognizes cholesterol and how it affects on other molecules participating in neurosteroidogenesis. StarD6 can take precedence over other molecules such as StAR, PBR and p450scc in excitotoxic brain injury and they might be hormonally regulated. There are no reports on the brain function or structure in StarD6 knockout animal model or in patients' brain and the brain may have StarD6-independent mechanisms of lipid/cholesterol sensing. Therefore, further studies are needed to elucidate the exact nuclear roles of StarD6 on neurosteroidogenesis with StAR in the nervous system. It could suggest new therapeutic strategy for epilepsy since neurosteroids act as autocrine or paracrine neuromodulators and the regulation of neurosteroidogenesis may have profound influences in the nervous system.

\section{Acknowledgment}

I am indebted to In Youb Chang (Chosun University, Republic of Korea) for critical reading of the chapter; Sa Sun Cho (Professor Emeritus, Seoul National University, Republic of Korea) for his helpful discussion. Anti-StarD6 antibody was a kind gift of Jaemog Soh (Chonnam National University, Republic of Korea).

\section{References}

Alpy, F. \& Tomasetto, C. (2005). Give lipids a START: the StAR-related lipid transfer (START) domain in mammals. Journal of Cell Science, Vol. 118, No. (Pt)13, (July 2005), pp. 2791-2801, ISSN 0021-9533 
Adibhatla, R.M. \& Hatcher, J.F. (2008). Altered lipid metabolism in brain injury and disorders. Subcellular Biochemistry, Vol. 49, (April 2008), pp. 241-268, ISSN 03060225

Baulieu, E.E. (1997). Neurosteroids: of the nervous system, by the nervous system, for the nervous system. Recent Progress in Hormone Research, Vol. 52, (January 1997), pp. 131, ISSN 0079-9963

Biagini, G.; Baldelli, E.; Longo, D.; Pradelli, L.; Zini, I.; Rogawski, M.A. \& Avoli, M. (2006). Endogenous neurosteroids modulate epileptogenesis in a model of temporal lobe epilepsy. Experimental Neurology, Vol. 201, No. 2, (October 2006), pp. 519-524, ISSN 0014-4886

Biagini, G.; Panuccio, G. \& Avoli, M. (2010). Neurosteroids and epilepsy. Current Opinion in Neurology, Vol. 23, No. 2, (April 2010), pp. 170-176, ISSN 1350-7540

Bose, H.S.; Whittal, R.M.; Ran, Y.; Bose, M.; Baker, B.Y. \& Miller, W.L. (2008). StAR-like activity and molten globule behavior of StARD6, a male germ-line protein. Biochemistry, Vol. 47, No. 8, (February 2008), pp. 2277-2288, ISSN 0006-2960

Brose, N. \& Rosenmund, C. (2002). Move over protein kinase C, you've got company: alternative cellular effectors of diacylglycerol and phorbol esters. Journal of Cell Science, Vol. 115, No. (Pt)23, (December 2002), pp. 4399-4411, ISSN 0021-9533

Cavazos, J.E.; Jones, S.M. \& Cross, D.J. (2004). Sprouting and synaptic reorganization in the subiculum and CA1 region of the hippocampus in acute and chronic models of partial-onset epilepsy. Neuroscience, Vol. 126, No. 3, (March 2004), pp. 677-688, ISSN 0306-4522

Chang, I.Y.; Shin, S.Y.; Kim, J.W.; Yu, J.M.; Kim, J.S.; Song, P.I. \& Yoon, S.P. (2007a). The changed immunolocalization of START-domain-containing 6 (StarD6) during the development of testes in rat perinatal hypothyroidism. Acta Histochemica, Vol. 109, No. 4, (August 2007), pp. 315-321, ISSN 0065-1281

Chang, I.Y.; Kim, J.H.; Hwang, G.; Song, P.I.; Song, R.J.; Kim, J.W. \& Yoon, S.P. (2007b). Immunohistochemical detection of StarD6 in the rat nervous system. Neuroreport, Vol. 18, No. 15, (October 2007), pp. 1615-1619, ISSN 0959-4965

Chang, I.Y.; Kim, J.K.; Lee, S.M.; Kim, J.N.; Soh, J.; Kim, J.W. \& Yoon, S.P. (2009). The changed immunoreactivity of StarD6 after pilocarpine-induced epilepsy. Neuroreport, Vol 20, No. 10, (July 2009), pp. 963-967, ISSN 0959-4965

Chang, I.Y.; Jeon, Y.J.; Jung, S.M.; Jang, Y.H.; Ahn, J.B.; Park, K.S. \& Yoon, S.P. (2010). Does the StarD6 mark the same as the StAR in the nervous system? Journal of Chemical Neuroanatomy, Vol. 40, No. 3, (November 2010), pp. 239-242, ISSN 0891-0618

Chia, W.J.; Jenner, A.M.; Farooqui, A.A. \& Ong, W.Y. (2008). Changes in cytochrome P4450 side chain cleavage expression in the rat hippocampus after kainite injury. Experimental Brain Research, Vol. 186, No. 1, (March 2008), pp. 143-149, ISSN 00144819

Chuang, Y.C.; Chen, S.D.; Liou, C.W.; Lin, T.K.; Chang, W.N.; Chan, S.H. \& Chang, A.Y. (2009). Contribution of nitric oxide, superoxide anion, and peroxynitrite to activation of mitochondrial apoptotic signaling in hippocampal CA3 subfield following experimental temporal lobe status epilepticus. Epilepsia, Vol. 50, No. 4, (June 2009), pp. 731-746, ISSN 0013-9580 
Clark, B.J.; Wells, J.; King, S.R. \& Stocco, D.M. (1994). The purification, cloning, and expression of a novel luteinizing hormone-induced mitochondrial protein in MA-10 mouse Leydig tumor cells. Characterization of the steroidogenic acute regulatory protein (StAR). Journal of Biological Chemistry, Vol. 269, No. 45, (November 1994), pp. 28314-28322, ISSN 0021-9258

Coulter, D.A.; Mclntyre, D.C. \& Lőőscher, W. (2002). Animal models of limbic epilepsies: what can they tell us? Brain Pathology, Vol. 12, No. 2, (April 2002), pp. 240-256, ISSN 1015-6305

Culmsee, C.; Bondada, S. \& Mattson, M.P. (2001). Hippocampal neurons of mice deficient in DNA-dependent protein kinase exhibit increased vulnerability to DNA damage, oxidative stress and excitotoxicity. Brain Research Molecular Brain Research, Vol. 87, No. 2, (March 2001), pp. 257-262, ISSN 0169-328X

di Michele, F.; Lekieffre, D.; Pasini, A.; Bernardi, G.; Benavides, J. \& Romeo, E. (2000). Increased neurosteroids synthesis after brain and spinal cord injury in rats. Neuroscience Letters, Vol. 284, No. 1-2, (April 2000), pp. 3-12, ISSN 0304-3940

Dinocourt, C.; Petanjek, Z.; Freund, T.F.; Ben-Ari, Y. \& Exclapez, M. (2003). Loss of interneurons innervating pyramidal cell dendrites and axon initial segments in the CA1 region of the hippocampus following pilocarpine-induced seizures. Journal of Comparative Neurology, Vol. 459, No. 4, (May 2003), pp. 407-425, ISSN 0021-9967

Fishel, M.L.; Vasko, M.R. \& Kelley, M.R. (2007). DNA repair in neurons: so if they don't divide what's to repair? Mutation Research, Vol. 614, No. 1-2, (January 2007), pp. 2436, ISSN 0027-5107

Furukawa, A.; Miyatake, A.; Ohnishi, T. \& Ichikawa, Y. (1998). Steroidogenic acute regulatory protein (StAR) transcripts constitutively expressed in adult rat central nervous system: colocalization of StAR, cytochrome P-450scc (CYP XIA1), and 3beta-hydroxysteroid dehydrogenase in the rat brain. Journal of Neurochemistry, Vol. 71, No. 6, (December 1998), pp. 2231-2238, ISSN 0022-3042

Garcia-Ovejero, D.; Veiga, S.; Garcia-Segura, L.M. \& DonCarlos, L.L. (2002). Glial expression of estrogen and androgen receptors after rat brain injury. Journal of Comparative Neurology, Vol. 450, No. 3, (August 2002), pp. 256-271, ISSN 0021-9967

Gomes, C.; Oh, S.D.; Kim, J.W.; Chun, S.Y.; Lee, K.S.; Kwon, H.B. \& Soh, J. (2005). Expression of the putative sterol binding protein Stard6 gene is male germ cell specific. Biology of Reproduction, Vol. 72, No. 3, (March 2005), pp. 651-658, ISSN 0006-3363

Jarrett, S.G.; Liang, L.P.; Hellier, J.L.; Staley, K.J. \& Patel, M. (2008). Mitochondrial DNA damage and impaired base excision repair during epileptogenesis. Neurobiology of Disease, Vol. 30, No. 1, (April 2008), pp. 130-138, ISSN 0969-9961

Joëls, M. (2009). Stress, the hippocampus, and epilepsy. Epilepsia, Vol. 50, No. 4, (April 2009), pp. 586-597, ISSN 0013-9580

Kajitani, K.; Yamaguchi, H.; Dan, Y.; Furuichi, M.; Kang, D. \& Nakabeppu, Y. (2006). MTH1, an oxidized purine nucleoside triphosphatase, suppresses the accumulation of oxidative damage of nucleic acids in the hippocampal microglia during kainiteinduced excitotoxicity. Journal of Neuroscience, Vol. 26, No. 6, (February 2006), pp. 1688-1698, ISSN 0270-6474 
Kim, H.J.; Park, C.H.; Roh, G.S.; Kang, S.S.; Cho, G.J. \& Choi, W.S. (2002). Changes of steroidogenic acute regulatory protein mRNA expression in postnatal rat development. Brain Research Developmental Brain Research, Vol. 139, No. 2, (December 2002), pp. 247-254, ISSN 0165-3806

King, S.R.; Manna, P.R.; Ishii, T.; Syapin, P.J.; Ginsberg, S.D.; Wilson, K.; Walsh, L.P.; Parker, K.L.; Stocco, D.M.; Smith, R.G. \& Lamb, D.J. (2002). An essential component in steroid synthesis, the steroidogenic acute regulatory protein, is expressed in discrete regions of the brain. Journal of Neuroscience, Vol. 22, No. 24, (December 2002), pp. 10613-10620, ISSN 0270-6474

Lavaque, E.; Sierra, A.; Azcoitia, I. \& Garcia-Segura, L.M. (2006). Steroidogenic acute regulatory protein in the brain. Neuroscience, Vol. 138, No. 3, (March 2006), pp. 741747, ISSN 0306-4522

Lavigne, P.; Najmanivich, R. \& Lehoux, J.G. (2010). Mammalian StAR-related lipid transfer (START) domains with specificity for cholesterol: structural conservation and mechanism of reversible binding. Subcellular Biochemistry, Vol. 51, (April 2010), pp. 425-437, ISSN 0306-0225

Ma, D.L.; Tang, Y.C.; Chen, P.M.; Chia, S.C.; Jiang, F.L.; Burgunder, J.M.; Lee, W.L. \& Tang, F.R. (2006). Reorganization of CA3 area of the mouse hippocampus after pilocarpine induced temporal lobe epilepsy with special reference to the CA3septum pathway. Journal of Neuroscience Research, Vol. 83, No. 2, (February 2006), pp. 318-331, ISSN 0360-4012

Martin, L.J. (2008). DNA damage and repair: relevance to mechanisms of neurodegeneration. Journal of Neuropathology and Experimental Neurology, Vol. 67, No. 5, (May 2008), pp. 377-387, ISSN 0022-3069

Mellon, S.H.; Griffin, L.D. \& Compagnone, N.A. (2001). Biosynthesis and action of neurosteroids. Brain Research Reviews, Vol. 37, No. 1-3, (November 2001), pp. 3-12, ISSN 0165-0173

Naegele, J.R. (2007). Neuroprotective strategies to avert seizure-induced neurodegeneration in epilepsy. Epilepsia, Vol. 48, No. Supplement s2, (April 2007), pp. 107-117, ISSN 0013-9580

Neema, M.; Navarro-Quiroga, I.; Chechlacz, M.; Gilliams-Francis, K.; Liu, J.; Lamonica, K.; Lin, S.L. \& Naegele, J.R. (2005). DNA damage and nonhomologous end joining in excitotoxicity: neuroprotective role of DNA-PKcs in kainic acid-induced seizures. Hippocampus, Vol. 15, No. 8, (October 2005), pp. 1057-1071, ISSN 1050-9631

Patte-Mensah, C.; Kappes, V.; Freund-Mercier, M.J.; Tsutsui, K. \& Mensah-Nyagan, A.G. (2003). Cellular distribution and bioactivity of the key steroidogenic enzyme, cytochrome P450 side chain cleavage, in sensory neural pathways. Journal of Neurochemistry, Vol. 86, No. 5, (September 2003), pp. 1233-1246, ISSN 0022-3042

Plassart-Schiess, E. \& Baulieu, E.E. (2001). Neurosteroids: recent findings. Brain Research Reveiws, Vol. 37, No. 1-3, (November 2001), pp. 133-140, ISSN 0165-0173

Ponting, C.P. \& Aravind, L. (1999). START: a lipid-binding domain in StAR, HD-ZIP and signaling proteins. Trends in Biochemical Sciences, Vol. 24, No. 4, (April 1999), pp. 130-132, ISSN 0968-0004

Quach, N.; Chan, T.; Lu, T.A.; Schreiber, S.S. \& Tan, Z. (2005). Induction of DNA repair proteins, Ref-1 and XRCC1, in adult rat brain following kainic acid-induced 
seizures. Brain Research, Vol. 1042, No. 2, (May 2005), pp. 236-240, ISSN 00068993

Rune, G.M. \& Frotscher, M. (2005). Neurosteroid synthesis in the hippocampus: role in synaptic plasticity. Neuroscience, Vol. 136, No. 3, (March 2005), pp. 833-842, ISSN 0306-4522

Sanon, N.; Carmant, L.; Emond, M.; Congar, P. \& Lacaille, J.C. (2005). Short-term effects of kainic acid on CA1 hippocampal interneurons differently vulnerable to excitotoxicity. Epilepsia, Vol. 46, No. 6, (June 2005), pp. 837-848, ISSN 0013-9580

Sierra, A.; Lavaque, E.; Perez-Martin, M.; Azcoitia, I.; Hales, D.B. \& Garcia-Segura, L.M. (2003). Steroidogenic acute regulatory protein in the rat brain: cellular distribution, developmental regulation and overexpression after injury. European Journal of Neuroscience, Vol. 18, No. 6, (September 2003), pp. 1458-1467, ISSN 0953$816 \mathrm{X}$

Sierra, A. (2004). Neurosteroids: the StAR protein in the brain. Journal of Neuroendocrinology, Vol. 16, No. 9, (September 2004), pp. 787-793, ISSN 0953-8194

Soccio, R.E.; Adams, R.M.; Romanowski, M.J.; Sehayek, E.; Burley, S.K. \& Breslow, J.L. (2002). The cholesterol-regulated StarD4 gene encodes a StAR-related lipid transfer protein with two closely related homologues, StarD5 and StarD6. Proceedings of the National Academy of Sciences of the United States of America, Vol. 99, No. 10, (May 2002), pp. 6943-6948, ISSN 0027-8424

Soccio, R.E. \& Breslow, J.L. (2003). StAR-related lipid transfer (START) proteins: mediators of intracellular lipid metabolism. Journal of Biological Chemistry, Vol. 278, No. 25, (June 2003), pp. 22183-22186, ISSN 0021-9258

Soccio, R.E.; Adams, R.M.; Maxwell, K.N. \& Breslow, J.L. (2005). Differential gene regulation of StarD4 and StarD5 cholesterol transfer proteins: activation of StarD4 by SREBP-2 and StarD5 by endoplasmic reticulum stress, Journal of Biological Chemistry, Vol. 280, No. 19, (May 2005), pp. 19410-19418, ISSN 0021-9258

Stocco, D.M. (2001). StAR protein and the regulation of the steroid hormone biosynthesis. Annual Review of Physiology, Vol. 63, (March 2001), pp. 193-213, ISSN 0066-4278

Stocco, D.M.; Wang, X.; Jo, Y. \& Mann, P.R. (2005). Multiple signaling pathways regulating steroidogenesis and star expression: more complicated than we thought. Molecular Endocrinology, Vol. 19, No. 11, (November 2005), pp. 2647-2659, ISSN 0888-8809

Tsutsui, K.; Sakamoto, H. \& Ukena, K. (2003). A novel aspect of the cerebellum: biosynthesis of neurosteroids in the Purkinje cell. Cerebellum, Vol. 2, No. 3, (June 2003), pp. 215222, ISSN 1473-4222

Webber, K.M.; Stocco, D.M.; Casadesus, G.; Bowen, R.L.; Atwood, C.S.; Previll, L.A.; Harris, P.L.; Zhu, X.; Perry, G. \& Smith, M.A. (2006). Steroidogenic acute regulatory protein (StAR): evidence of gonadotrophin-induced steroidogenesis in Alzheimer disease. Molecular neurodegeneration, Vol. 1, (October 2006), pp. 14, ISSN 17501326

Wittner, L.; Eross, L.; Czirjak, S.; Halasz, P.; Freund, T.F. \& Magloczky, Z. (2005). Surviving CA1 pyramidal cells receive intact perisomatic inhibitory input in the human epileptic hippocampus. Brain, Vol. 128, No. (Pt)1, (January 2005), pp. 138-152, ISSN 0006-8950 
Wojtal, K.; Trojnar, M.K. \& Czuczwar S.J. (2006). Endogenous neuroprotective factors: neurosteroids. Pharmacological Reports, Vol. 58, No. 3, (May-June 2006), pp. 335-340, ISSN 1734-1140.

Zhang, S.; Khanna, S. \& Tang, F.R. (2009). Patterns of hippocampal neuronal loss and axon reorganization of the dentate gyrus in the mouse pilocarpine model of temporal lobe epilepsy. Journal of Neuroscience Research, Vol. 87, No. 5, (April 2009), pp. 11351149, ISSN 0360-4012 


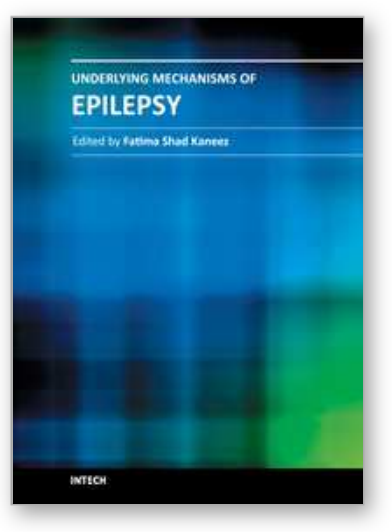

\author{
Underlying Mechanisms of Epilepsy \\ Edited by Prof. Fatima Shad Kaneez
}

ISBN 978-953-307-765-9

Hard cover, 354 pages

Publisher InTech

Published online 26, September, 2011

Published in print edition September, 2011

This book is a very provocative and interesting addition to the literature on Epilepsy. It offers a lot of appealing and stimulating work to offer food of thought to the readers from different disciplines. Around $5 \%$ of the total world population have seizures but only $0.9 \%$ is diagnosed with epilepsy, so it is very important to understand the differences between seizures and epilepsy, and also to identify the factors responsible for its etiology so as to have more effective therapeutic regime. In this book we have twenty chapters ranging from causes and underlying mechanisms to the treatment and side effects of epilepsy. This book contains a variety of chapters which will stimulate the readers to think about the complex interplay of epigenetics and epilepsy.

\title{
How to reference
}

In order to correctly reference this scholarly work, feel free to copy and paste the following:

Sang Pil Yoon (2011). START Proteins in Epilepsy, Underlying Mechanisms of Epilepsy, Prof. Fatima Shad Kaneez (Ed.), ISBN: 978-953-307-765-9, InTech, Available from:

http://www.intechopen.com/books/underlying-mechanisms-of-epilepsy/start-proteins-in-epilepsy

\section{INTECH}

open science | open minds

\section{InTech Europe}

University Campus STeP Ri

Slavka Krautzeka 83/A

51000 Rijeka, Croatia

Phone: +385 (51) 770447

Fax: +385 (51) 686166

www.intechopen.com

\section{InTech China}

Unit 405, Office Block, Hotel Equatorial Shanghai

No.65, Yan An Road (West), Shanghai, 200040, China

中国上海市延安西路65号上海国际贵都大饭店办公楼 405 单元

Phone: +86-21-62489820

Fax: +86-21-62489821 
(C) 2011 The Author(s). Licensee IntechOpen. This chapter is distributed under the terms of the Creative Commons Attribution-NonCommercialShareAlike-3.0 License, which permits use, distribution and reproduction for non-commercial purposes, provided the original is properly cited and derivative works building on this content are distributed under the same license. 